\title{
Changes and Significance of Serum sB7-H3 and Cytokines in Children with Mycoplasma pneumonae Pneumonia
}

\author{
Peng $\mathrm{Wu}^{1}$ and Jian Wang ${ }^{2}$ \\ ${ }^{1}$ Department of Clinical Laboratory, Renmin Hospital of Wuhan University, Wuhan, China \\ ${ }^{2}$ Department of Clinical Laboratory, Union Hospital, Tongji Medical College, Huazhong University of Science and Technology, Wuhan, China
}

\begin{abstract}
Objective: To explore the relationship between serum sB7-H3 and cytokines (TNF- $\alpha$, IL-1 $\beta$ and IL-6) and to evaluate the development of Mycoplasma pneumonae pneumonia (MPP) through analysis of the expression levels of above indices in serum of children with MPP.

Study Design: An experimental study.

Place and Duration of Study: Department of Clinical Laboratory, Renmin Hospital of Wuhan University, China, from January 2018 to August 2019.

Methodology: One hundred and eight children with MPP were divided into severe MPP group (53 cases) and mild MPP group (55 cases) according to children's condition. Fifty children who received hernia or selective operation due to redundant prepuce were included in control group. Serum sB7-H3, TNF- $\alpha$, IL-1 $\beta$ and IL-6 were compared.

Results: Serum sB7-H3, TNF- $\alpha$, IL-1 $\beta$, and IL-6 levels in MPP group were higher than those in control group (all $p<0.001$ ); above indices in severe MPP group were higher than those in mild MPP group (all $p<0.001)$. Pearson's linear correlation analysis results revealed that sB7-H3 had positive correlation with TNF- $\alpha$, IL-1 $\beta$, and IL-6 in MPP group $(r=0.986, p<0.001 ; r=0.987, p<0.001 ;$ and $r=0.991, p<0.001$, respectively).

Conclusion: Detection of SB7-H3, TNF- $\alpha$, IL-1 $\beta$ and IL-6 levels may be conducive to early diagnosis of MPP and the judgement of the severity of this disease.
\end{abstract}

Key Words: Mycoplasma pneumoniae pneumonia (MPP), Children, sB7-H3, Cytokines.

How to cite this article: Wu P, Wang J. Changes and significance of serum sB7-H3 and cytokines in children with mycoplasma pneumonae pneumonia. J Coll Physicians Surg Pak 2020; 30(3):268-271.

\section{INTRODUCTION}

Mycoplasma pneumonae pneumonia (MPP) as one of the primary community-acquired pneumonia may cause extrapulmonary complications easily and result in severe pneumonia, thereby seriously threatening children's health. ${ }^{1}$ Currently, the pathogenesis of MPP is widely attributed to the fact that the release of certain inflammatory mediators and cytokines under the promotion of Mycoplasma pneumoniae causes body immune regulation dysfunction, thus leading to body immune injury and inducing MPP., 2,3 The costimulatory molecule soluble B7 homolog $3(\mathrm{sB} 7-\mathrm{H} 3)$ is a member of the chemokine family that will exacerbate the autoimmune injury of lung epithelial cells by inducing the activation of chemokines and complement components, and accelerate the progress of MPP. ${ }^{4}$ TNF- $\alpha$ is an important inflammatory factor mainly produced by macrophages and monocytes with close correlation to

Correspondence to: Dr. Wang, Department of Clinical Laboratory, Union Hospital, Tongji Medical College, Huazhong University of Science and Technology, Wuhan 430022, China

E-mail:dwpcsr@163.com

Received: December 11, 2019; Revised: February 14, 2020;

Accepted: February 14, 2020. inflammation and infection. ${ }^{5}$ Interleukin- $1 \beta$ (IL-1 $\beta$ ) is an important inflammatory factor dually sourced from peripheral and central nervous system that can induce the generation and release of IL-8 and other inflammatory factors. ${ }^{6}$ IL-6 is an important immunoregulatory factor which participates in the pathological process of the lungs and humoral immune regulation. It often occurs during the acute period of inflammatory reaction and the lung infection degree can be manifested by its level. ${ }^{7,8}$ The specific process of changes in sB7-H3 and cytokines (TNF- $\alpha, \mathrm{IL}-1 \beta$ and IL-6) at onset of MPP is unclear yet.

The aim of this study was to explore relationship between serum sB7-H3 and cytokines (TNF- $\alpha$, IL-1 $\beta$ and $(\mathrm{L}-6)$ and to evaluate the development of MPP through analysis of the expression levels of above indices in serum of children with MPP.

\section{METHODOLOGY}

The experimental study was carried out at Department of Clinical Laboratory, Renmin Hospital of Wuhan University, China, from January 2018 to August 2019. The study was conducted after obtaining informed consent from children's family members upon the approval of the Hospital Medical Ethics Committee. One 
hundred and eight children with MPP (MPP group) were selected and divided into severe MPP group (53 cases) and mild MPP group (55 cases), according to children's condition. The MPP diagnostic criteria: patients with clinical manifestations of fever, cough, dyspnea, and lung rale; abnormal changes in unilateral or bilateral chest were detected by imaging examination; the presence of positive MP-specific IgM antibody; no other type of pathogen infection was detected at the initial stage of disease course. Inclusion criteria were: children who met MPP diagnostic criteria without being treated with macrolide antibiotics since the onset of the disease; without immune disease; without unexplained long-term fever or joint swelling and pain; and those without other endoscopic diseases, whose parents accepted the study with informed consent. Exclusion criteria were: children with congenital immune diseases; other pathogen infections recently; those with allergic constitution.

Children were labelled severe MPP if they have two of these condition; body temperature of exceeding $38.5^{\circ} \mathrm{C}$ continuously or no improvement or even worsened condition as shown in chest radiography after being treated by macrolides for more than 7 days; large shadows above the lung or lung segment as shown in chest radiograph; obvious symptoms of anhelation or tachycardia; pulmonary atelectasis, lung abscess, pleural effusion and other complications; hypoxemia. Heart failure and other extrapulmonary complications. The criteria for mild MPP were no dyspnea, no intrapulmonary and extrapulmonary complications after being treated with macrolides for 7 days.

In addition, 50 children who received hernia or selective operation due to redundant prepuce from January 2018 to August 2019, were included in control group: Inclusive criteria in control group were: children with no skin, respiratory tract, digestive system, urinary system and other infection symptoms over the past one month, and children without being diagnosed with immune diseases in the past. Children with long-term fever, joint swelling and pain, children with the medical history of recent or long-term use of glucocorticoids, children using immunoglobulins, interferons and other immuno-modulators and immunosuppressants over the past two months were excluded.

The venous blood of MPP children in the acute period at 24 hours after admission was taken for centrifugation collection of serum. After admission and diagnosis, MPP pediatric patients were divided into severe MPP group and mild MPP group according to their condition. The serum of the subjects was collected. One-time serum of children in control group was retained. The expression serum levels of sB7-H3 and cytokines TNF- $\alpha, \mathrm{IL}-1 \beta$ and IL-6 were detected by enzyme-linked immunosorbent assay.

Data was analysed by SPSS 25.0. Measurement data were denoted as mean $\pm S D$, and independent sample t-test was used. Count data were denoted as $\mathrm{n}(\%)$. Pearson's linear correlation analysis was used. Origin 9.0 was used as the correlation analysis chart. The $p<0.05$ was considered as the difference with statistical significance.

\section{RESULTS}

Among 108 children in MPP group, 56 cases (51.85\%) were boys and 52 cases $(48.15 \%)$ were girls, aged l-13 $(5.71 \pm 1.95)$ years, with onset time of 1-7 $(3.53 \pm 1.44)$ days. Among the 55 children with mild MPP, there were $30(54.55 \%)$ boys and 25 girls $(45.45 \%)$ between the age of 1-13 (5.96 \pm 1.75$)$ years. Among the 53 children patients with severe MPP, 26 cases $(49.06 \%)$ were boys and 27 cases $(50.94 \%)$ were girls, aged of $1-13$ (5.58 \pm 1.81 ) years. Among 50 subjects in control group, there were 26 boys $(52.00 \%)$ and, 24 girls $(48.00 \%)$ between the age of $\mathrm{I}-13(5.84 \pm 2.47)$ years.

Serum sB7-H3, TNF- $\alpha$, IL-1 $\beta$, and IL-6 levels in MPP group were higher than those in control group (all $\mathrm{p}<0.001$, Table I). Serum sB7-H3, TNF- $\alpha$, IL-1 $\beta$, and IL-6 levels in severe MPP group were higher than those in mild MPP group (all $p<0.001$, Table II). Pearson's linear correlation analysis results showed that the serum level of sB7-H3 had positive correlation with TNF- $\alpha, \mathrm{IL}-1 \beta$ and IL-6 in MPP group ( $r=0.986, p<0.001 ; r=0.987, p<0.001$; and $r=0.991, p<0.001$ respectively, Figure 1$)$.

Table I: Comparison of sB7-H3, TNF- $\alpha$, IL-1 $\beta$, IL-6 between MPP group and control group.

\begin{tabular}{lccc}
\hline Indices & $\begin{array}{c}\text { MPP group } \\
(\mathrm{n}=108)\end{array}$ & $\begin{array}{c}\text { Control group } \\
(\mathrm{n}=50)\end{array}$ & p-value \\
\hline $\mathrm{sB} 7-\mathrm{H} 3(\mu \mathrm{g} / \mathrm{L})$ & $8.58 \pm 0.63$ & $5.47 \pm 0.46$ & $<0.001$ \\
\hline $\mathrm{TNF}-\alpha(\mathrm{ng} / \mathrm{L})$ & $71.37 \pm 1.68$ & $21.84 \pm 1.25$ & $<0.001$ \\
\hline $\mathrm{IL}-1 \beta(\mathrm{ng} / \mathrm{L})$ & $191.30 \pm 3.15$ & $28.89 \pm 2.54$ & $<0.001$ \\
\hline $\mathrm{IL}-6(\mathrm{ng} / \mathrm{mL})$ & $2.74 \pm 0.10$ & $0.37 \pm 0.06$ & $<0.001$ \\
\hline
\end{tabular}

Table II: Comparison of sB7-H3, TNF- $\alpha$, IL-1 $1 \beta$, IL-6 between mild MPP group and severe MPP group.

\begin{tabular}{lccc}
\hline Indices & $\begin{array}{c}\text { Mild MPP group } \\
(\mathrm{n}=55)\end{array}$ & $\begin{array}{c}\text { Severe MPP group } \\
(\mathrm{n}=55)\end{array}$ & p-value \\
\hline $\mathrm{sB} 7-\mathrm{H} 3(\mu \mathrm{g} / \mathrm{L})$ & $6.56 \pm 0.85$ & $11.04 \pm 1.22$ & $<0.001$ \\
\hline $\mathrm{TNF}-\alpha(\mathrm{ng} / \mathrm{L})$ & $68.70 \pm 2.01$ & $116.67 \pm 3.44$ & $<0.001$ \\
\hline $\mathrm{IL}-1 \beta(\mathrm{ng} / \mathrm{L})$ & $181.53 \pm 3.17$ & $241.32 \pm 3.69$ & $<0.001$ \\
\hline $\mathrm{IL}-6(\mathrm{ng} / \mathrm{mL})$ & $2.04 \pm 0.12$ & $3.88 \pm 0.61$ & $<0.001$ \\
\hline
\end{tabular}

\section{DISCUSSION}

Costimulatory molecules synergistic or antagonistic with cytokines can jointly participate in MPP pathogenic process. ${ }^{9}$ sB7-H3 can participate in body immune regulation. As it has been reported that $\mathrm{sB} 7-\mathrm{H} 3$ can upregulating the expression of certain cytokines, which is correlated with the duration of MPP disease course. ${ }^{10}$ It has also been founded by some studies that the expression of $\mathrm{sB} 7-\mathrm{H} 3$ in serum of patients under 

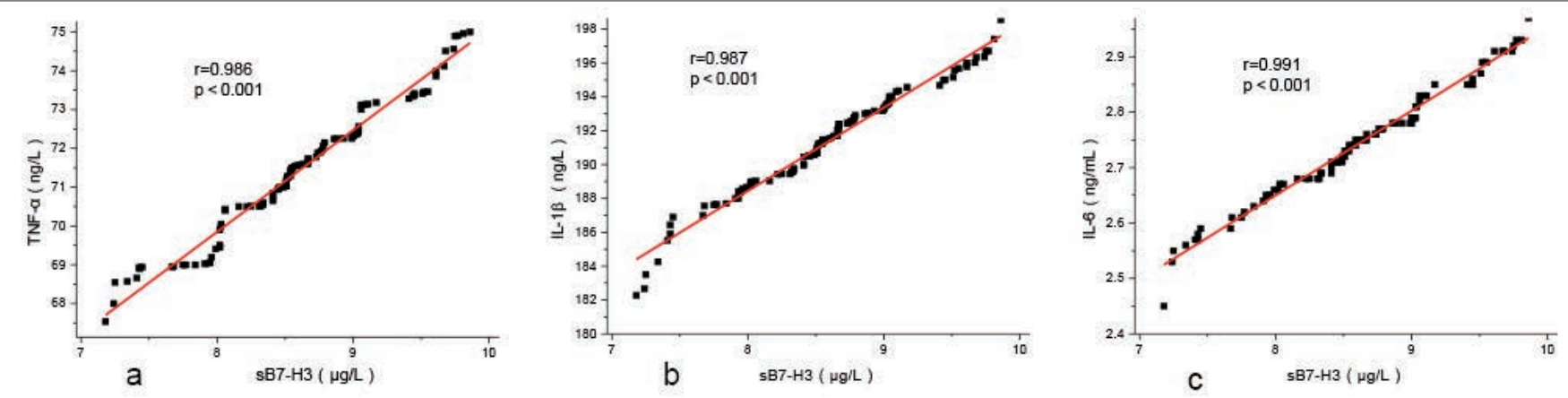

Figure 1: Correlation analysis between sB7-H3 and TNF- $\alpha$ (a), IL-1 $\beta$ (b) and IL-6 (c) in MPP children.

mycoplasma pneumonae infection was significantly upregulated and the concentration was significantly increased. ${ }^{11}$

In this study, serum sB7-H3 levels in MPP group were higher than those in control group, which were correlated with the severity of MPP.

A large amount of TNF- $\alpha$ will be produced by macrophage after being induced by mycoplasma pneumoniae, leading to lung damage in situ. In addition, a large number of TNF- $\alpha$ will enter the blood circulation and cause extrapulmonary tissue injury. It has also been found by one study that TNF- $\alpha$ showed high expression in bronchoalveolar lavage fluid in patients with refractory mycoplasma pneumonae pneumonia. ${ }^{12}$ In this study, it was found that serum TNF-a levels in MPP group were higher than those in control group and correlated with the severity of MPP.

$\mathrm{IL}-1 \beta$ is regarded as a multifunctional cytokine, which can activate $T$ cells. It has been indicated that lung inflammatory reaction of bovine infectious pleuropneumonia could increase the IL-1 $\beta$ factor expression. ${ }^{13}$ In this study, it was found that IL-1 $\beta$ in the MPP group increased significantly compared to that in the control group, and also significantly increased in the mild and severe groups of MPP, suggesting that IL-1 $\beta$ participated in the development of MPP. This generally accords with the researches and reports by Lieberman et al. ${ }^{14}$

IL-6, which is generated by activated macrophages, monocytes, $T$ cells has a low concentration in normal serum. ${ }^{15}$ During the acute period of mycoplasma infection, strengthening the release and synthesis of inflammatory cytokines, and the generation of autoantibodies by inducing the differentiation of $T$ cells can cause autoimmunity to occur. The degree of increase is closely related to the occurrence and development of infection. ${ }^{16}$ IL-6 as an important endogenous pyrogen will give rise to acute fever. ${ }^{17}$ According to one study, interleukin-6 may participate in the process of central nervous system inflammation, caused by mycoplasma pneumonae. $^{18}$
This study showed that serum IL-6 level in MPP group was higher than that in control group. The difference in serum IL- 6 between the mild MPP group and the severe group also had statistical significance. Therefore, the important role and clinical significance of IL-6 in the pathogenic process of mycoplasma pneumonae should be highly valued.

According to Pearson's linear correlation analysis results, serum sB7-H3 level in MPP group was positively correlated with TNF- $\alpha$, IL-1 $\beta$, and IL-6, suggesting that B7-H3 could promote the generation of cytokines TNF- $\alpha$, $\mathrm{IL}-1 \beta$, and IL- 6 by playing a dual-directional regulating role in balancing Th1 type cytokines (TNF- $\alpha$, IL-1 $\beta$, etc.) and Th2 type cytokines (IL-6, etc.). ${ }^{19-21}$ Expression levels of sB7-H3 and TNF- $\alpha$, IL-1 $\beta$, and IL- 6 were associated with severity of MPP disease. By monitoring the changes in the level of the above indices during the whole course of children patients with MPP, it is feasible to analyse and estimate the progress and prognosis of children patients with MPP.

\section{CONCLUSION}

SB7-H3, TNF- $\alpha$, IL-1 $\beta$ and IL-6 increased significantly in serum of children patients with MPP. Detection of above indices may be conducive to early diagnosis of MPP and the judgement of the severity of this disease.

\section{ETHICAL APPROVAL:}

This study was approved by Renmin Hospital of Wuhan University Medical Ethics Committee, China.

\section{PATIENTS' CONSENT:}

Informed consents were obtained from parents of children.

\section{CONFLICT OF INTEREST:}

Authors declared no conflict of interest.

\section{AUTHORS' CONTRIBUTION:}

PW: Data collection, statistics, and discussion. JW: Supervision, revision, and final approval. 


\section{REFERENCES}

1. Okumura T, Kawada JI, Tanaka M, Narita K, Ishiguro T, Hirayama $\mathrm{Y}$, et al. Comparison of high-dose and low-dose corticosteroid therapy for refractory mycoplasma pneumonae pneumonia in children. $J$ Infect Chemother 2019; 25:346-50.

2. Ranjbar R, Halaji M. Epidemiology of mycoplasma pneumonae prevalence in iranian patients: A systematic review and metaanalysis. J Med Microbiol 2019; 68:1614-21.

3. Li Q, Li ZY, Zhang J, Guo WN, Xu XM, Sun FX, et al. Xiyanping plus azithromycin chemotherapy in pediatric patients with mycoplasma pneumonae pneumonia: A systematic review and meta-analysis of efficacy and safety. Evid Based Complement Alternat Med 2019; 2019:2346583.

4. Chen ZR, Zhang GB, Wang YQ, Yan YD, Zhou WF, Zhu CH, et al. Soluble B7-H3 elevations in hospitalized children with mycoplasma pneumonae pneumonia. Diagn Microbiol Infect Dis 2013; 77:362-6.

5. Ferreiro L, Lado-Baleato Ó, Suárez-Antelo J, Toubes ME, San José ME, Lama A, et al. Diagnosis of infectious pleural effusion using predictive models based on pleural fluid biomarkers. Valdés L Ann Thorac Med 2019; 14:254-63.

6. Hewett SJ, Jackman NA, Claycomb RJ. Interleukin-1ß in central nervous system injury and repair. Eur $J$ Neurodegener Dis 2012; 1:195-211.

7. Rawlings SA, Heldt S, Prattes J, Eigl S, Jenks JD, Flick H, et al. Using interleukin 6 and 8 in blood and bronchoalveolar lavage fluid to predict survival in hematological malignancy patients with suspected pulmonary mold infection. Front Immunol 2019; 10:1798.

8. Dalvi SM, Ramraje NN, Patil VW, Hegde R, Yeram N. Study of IL-6 and vitamin D3 in patients of pulmonary tuberculosis. Indian J Tuberc 2019; 66:337-45.

9. Li QL, Wu YY, Sun HM, Gu WJ, Zhang XX, Wang MJ, et al. The role of miR-29c/B7-H3/Th17 axis in children with mycoplasma pneumonae pneumonia. Ital J Pediatr 2019; 45:61.

10. Chen Z, Zhao X, Zhang X, Zhang G, Sun H, Jiang W, et al. Increased concentrations of soluble B7-H3 and interleukin 36 in bronchoalveolar lavage fluid of Children with mycoplasma pneumonae pneumonia. BMC Infect Dis 2016; 16:212.

11. Xu Y, Yu L, Hao C, Wang Y, Zhu C, Ji W, et al. Plasma soluble $\mathrm{B} 7-\mathrm{H} 3$ levels for severity evaluation in pediatric patients with mycoplasma pneumonae pneumonia. Int Immunopharmacol 2019; 73:163-71.

12. Li G, Fan L, Wang Y, Huang L, Wang M, Zhu C, et al. High coexpression of TNF- $\alpha$ and CARDS toxin is a good predictor for refractory mycoplasma pneumonae pneumonia. Mol Med 2019; 25:38.

13. Sterner-Kock A, Haider W, Sacchini F, Liljander A, Meens J, Poole $\mathrm{J}$, et al. Morphological characterization and immunohistochemical detection of the proinflammatory cytokines $\mathrm{IL}-1 \beta$, IL-17A, and TNF- $\alpha$ in lung lesions associated with contagious bovine pleuropneumonia. Trop Anim Health Prod 2016; 48:569-76.

14. Lieberman D, Livnat S, Schlaeffer F, Porath A, Horowitz S, Levy R. IL-1beta and IL-6 in community-acquired pneumonia: bacteremic pneumococcal pneumonia versus mycoplasma pneumonae pneumonia. Infection 1997; 25:90-4.

15. Chonov DC, Ignatova MMK, Ananiev JR, Gulubova MV. IL-6 activities in the tumour microenvironment. Part 1. Open Access Maced J Med Sci 2019; 7:2391-8.

16. Buck C, Bundschu J, Gallati H, Bartmann P, Pohlandt F. Interleukin-6: A sensitive parameter for the early diagnosis of neonatal bacterial infection. Pediatrics 1994; 93:54-8.

17. Akata Y, Morimoto A, Long NC, Murakami N. Fever and acutephase response induced in rabbits by intravenous and intracerebroventricular injection of interleukin-6. Cytokine 1991; 3: 199-203.

18. Narita M, Tanaka H, Togashi T, Abe S. Cytokines involved in CNS manifestations caused by mycoplasma pneumonae. Pediatric Neurology 2005; 33:105-9.

19. Yoon BR, Chung YH, Yoo SJ, Kawara K, Kim J, Yoo IS, et al. Preferential induction of the $\mathrm{T}$ cell auxiliary signaling molecule b7-h3 on synovial monocytes in rheumatoid arthritis. $J$ Biol Chem 2016; 29:4048-57.

20. Inamura K, Amori G, Yuasa T, Yamamoto S, Yonese J, Ishikawa Y. Relationship of B7-H3 expression in tumor cells and tumor vasculature with FOXP3+ regulatory T cells in renal cell carcinoma. Cancer Manag Res 2019; 11:7021-30.

21. Zhang G, Huang $H$, Zhu $Y, Y u$ G, Gao X, Xu Y, et al. A novel subset of B7-H3+CD14+HLA-DR-/low myeloid-derived suppressor cells are associated with progression of human NSCLC. Oncoimmunology 2015; 4:e97716. 